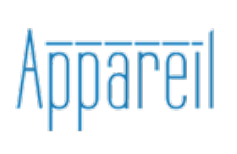

Appareil

11 | 2013

L'espace et l'architecture: état des lieux

\title{
La « révolution de l'espace » et l'architecture comme « réalisation de la philosophie » dans Écumes de Sloterdijk
}

Jean-Hugues Barthélémy

\section{(2) OpenEdition \\ Journals}

Édition électronique

URL : http://journals.openedition.org/appareil/1767

DOI : 10.4000/appareil. 1767

ISSN : 2101-0714

Éditeur

MSH Paris Nord

Référence électronique

Jean-Hugues Barthélémy, «La « révolution de l'espace » et l'architecture comme « réalisation de la philosophie » dans Écumes de Sloterdijk », Appareil [En ligne], 11 | 2013, mis en ligne le 26 septembre 2013, consulté le 30 juillet 2020. URL : http://journals.openedition.org/appareil/1767 ; DOI : https:// doi.org/10.4000/appareil.1767

Ce document a été généré automatiquement le 30 juillet 2020.

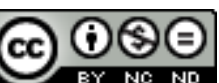

Appareil est mis à disposition selon les termes de la Licence Creative Commons Attribution - Pas d'Utilisation Commerciale - Pas de Modification 4.0 International. 


\section{La « révolution de l'espace » et l'architecture comme « réalisation de la philosophie » dans Écumes de Sloterdijk}

Jean-Hugues Barthélémy

\section{Les ambiguiités d'une pensée : autour de l'« affaire Sloterdijk »}

1 Peter Sloterdijk est, en Allemagne mais aussi en France, à la fois un grand intellectuel assez médiatisé et l'homme d'un scandale : celui créé par le désormais célèbre opuscule intitulé Règles pour le parc humain, qui est en réalité le texte d'une conférence. Cette dernière se voulait, ainsi que l'indique du reste son sous-titre, une " Lettre en réponse à la Lettre sur l'humanisme de Heidegger ", mais la reprise par Sloterdijk de certains concepts et de certaines thématiques de Heidegger - auteur désormais honni en Allemagne -, ainsi que la présence de Platon et de Nietzsche aux côtés de Heidegger dans le texte, ont laissé croire aux lecteurs qui n'étaient pas vraiment entrés dans ce texte que les expressions de " parc humain » et d'« élevage de soi-même » utilisées par Sloterdijk relevaient d'un élitisme heideggériano-platonicien. Bien sûr, il suffit de le lire attentivement pour comprendre que Sloterdijk vise bien plutôt à soutenir la thèse suivante :

Nous avons quitté l'ère de l'humanisme des temps modernes, considéré comme un modèle scolaire et éducatif, parce que l'on ne peut plus maintenir l'illusion selon laquelle les grandes structures politiques et économiques pourraient être organisées selon le modèle amiable de la société littéraire. ${ }^{1}$

Une telle thèse, formulée en 1999, est aujourd'hui devenue une banalité et ne peut redevenir profonde qu'à la condition qu'on l'inscrive dans un débat avec Foucault pour lui faire dire autre chose et davantage que ce qu'elle dit: le «biopouvoir » qui, jusque 
dans les écoles et sous forme de discipline des corps, a accompagné l'humanisme de la Modernité - comme humanisme fondé sur les "Humanités » littéraires -, a pris la forme tout à fait nouvelle de ce que Sloterdijk nomme les " médias de la désinhibition " ${ }^{2}$ et que Stiegler, en France cette fois, nomme le "psycho-pouvoir» des «industries culturelles », en le(s)quel(s) s'effondre la République des Lettres, et que l'on ne pourra surmonter qu'en acceptant de penser l'homme comme cet animal "néoténique ", dit Sloterdijk, qui est fait par la technique autant qu'il la fait. Sur ce dernier point, qui marque certainement la grande co-ïncidence entre le Stiegler de 1994 et le Sloterdijk de 1999-2000, ce dernier écrit :

en réalité, l'homme, conçu comme créature d'une espèce et comme matrice de chances d'individualisation, est une dimension qui n'existe pas, qui ne peut exister dans la nature; elle ne s'est engendrée d'elle-même que sous l'effet rétroactif de prototechniques spontanées, au cours de très longs processus de formation ayant une tendance contre-naturelle. ${ }^{3}$

3 En d'autres termes, ce que Sloterdijk nomme les "anthropotechniques", comme techniques du contrôle de l'homme qui se sont radicalisées - dit-il-avec les technologies génétiques, possède sa condition de possibilité dans le fait extrêmement lointain de ce que Sloterdijk nomme la "révolution anthropogénétique ", c'est-à-dire cette ouverture au monde d'un primate qui se dresse pour manier des objets et en fabriquer. Sloterdijk révèle alors qu'en réalité son concept d' "anthropotechniques » désigne à la fois le conditionné contemporain - les technologies appliquées à l'homme et le conditionnant originel - l'homme comme être prothétique :
Je veux essayer de montrer dans les lignes qui viennent que la méditation heideggérienne sur l'extase existentielle a aussi une signification pour la compréhension de la crise actuelle dans la définition biologique de l'homme par soi-même - dans cette crise qui affecte les modes d'intervention de l'homme sur l'homme, pour lesquels j'ai introduit dans mon discours prononcé à Bâle et à Elmau, Règles pour le parc humain, l'expression " anthropotechnique ». Très récemment, au cours d'un vaste débat, on a compris ce terme comme le synonyme du concept d'une biotechnologie humaine effectuant une planification stratégique [...]. En vérité, l'expression "anthropotechnique » désigne un théorème philosophique et anthropologique de base selon lequel l'homme lui-même est fondamentalement un produit et ne peut donc être compris que si l'on se penche, dans un esprit analytique, sur son mode de production. ${ }^{4}$

Comprendre cette "révolution anthropogénétique ", c'est-à-dire cet avènement bouleversant de l'humain sur Terre, comme « prothéticité » (Stiegler) de l'être-homme, c'est se donner les moyens de penser l'homme par-delà l'humanisme des Modernes, qui en effet ne pensait pas la genèse de l'homme mais le coupait radicalement de l'animal en faisant de la « Raison » l'essence du premier. Par là même, c'est se donner les moyens de comprendre le lien intime entre ce devenir-homme originel et les menaces techniques qui pèsent aujourd'hui sur l'homme. Par là enfin, c'est se donner quelques réelles chances de résister aux effets pervers des "médias de la désinhibition" (Sloterdijk) et de la culture de masse que ces industries « culturelles» organisent $^{5}$. En ce point, l'apport incontestable de Stiegler aux interrogations de Sloterdijk est la démarche subtile de la " pharmacologie ", qui tente de penser la technique comme étant à la fois le poison et le remède, parce qu'elle est ce qui fait l'homme.

On remarquera cependant l'ambiguïté de la thèse commune à Stiegler et Sloterdijk : d'un côté il s'agit de penser la genèse de l'homme, mais d'un autre côté la " prothéticité » reste un propre de l'homme en tant que ce dernier, contrairement aux 
autres espèces, n'est pas fait par la nature. J'ai discuté cette thèse à l'occasion d'un dialogue avec Stiegler concernant la question, plus générale et plus fondamentale, de la réfutation de l'idéalisme au sein de l'héritage français de Simondon ${ }^{6}$.

Quelle que soit à mes yeux l'importance de cette réserve concernant les constructions philosophiques de Sloterdijk et de Stiegler, un premier enseignement est en tout cas qu'il semble possible de rapprocher ces deux penseurs, mais à condition de prêter à Sloterdijk une intention d'Aufklärer qu'il n'a peut-être pas jusqu'au bout, parce qu'une telle intention ne peut survivre aujourd'hui que chez celui qui pense la différence subtile entre l'idée des "Lumières" et ce qu'a été l'humanisme des Modernes. Rappelons donc ici que les Lumières sont le penser par soi-même et l'Encyclopédisme toujours ouvert au devenir des sciences -, tandis que l'humanisme des Modernes a prétendu à un Savoir philosophique fondé sur la raison comprise comme "essence de l'homme». J'ai produit ailleurs l'explicitation d'une telle différence subtile, en montrant - avec et après Simondon - que l'Encyclopédisme, en tant qu'il s'oppose au Savoir figé, est le seul trait par lequel les Lumières et même l'Humanisme peuvent se dépasser eux-mêmes et prendre aujourd'hui une nouvelle forme, tout à fait inédite - et parfaitement irréductible à l'humanisme des Modernes ${ }^{7}$.

Sloterdijk, lui, dit à terme son non-engagement :

8 a/ il distingue d'abord entre les «médias en forme d'appareils, qui transmettent des programmes, et des médias personnels, c'est-à-dire des gens dotés d'une certaine perméabilité, qui transmettent les missions d'une époque ou les tensions de leur temps $»^{8}$;

9 b/ cette dernière distinction - interne au second concept de média - entre ceux qui transmettent « les missions d'une époque » et ceux qui transmettent «les tensions de leur temps » est également formulée, bien que différemment, dans cette thèse précoce des entretiens avec Hans-Jürgen Heinrichs : "après les Lumières, si on ne les a pas contournées, il ne peut plus exister de medias directement religieux, mais sans doute les medias d'une ambiance historique, ou les medias d'une urgence ${ }^{9}$;

c/ Sloterdijk applique finalement à lui-même l'appellation de "média d'ambiance " plutôt que celle de "média d'urgence » - qui s'appliquerait bien plutôt à Stiegler lorsqu'après avoir distingué entre les médias-appareils et les médias-personnalités il écrit :

Si l'on rassemble ces deux concepts de media, et si on les applique à son propre rôle, on peut en arriver, en quelque sorte, à se soupçonner soi-même d'être un appareil. La théorie récente de la littérature et des médias parle de l'auteur comme d'une machine à écrire neurologique, et cela correspond parfois à l'expérience que l'on a de soi-même quand on est en position d'auteur. Pour ma part, je préférerais me comparer à un piano qui se met subitement à jouer tout seul. Un piano automatique de l'esprit du temps. J'enregistre facilement les ambiances, mais je trie avec une assez grande sévérité. ${ }^{10}$

11 «Enregistrer les ambiances» comme le ferait «un piano automatique de l'esprit du temps ", voilà la tâche que s'attribue Sloterdijk, qui ne prétend hériter des Lumières qu'en tant que celles-ci, selon lui, «ont quelque chose à voir avec la clarification de l'humeur sociale et individuelle». Étrange réduction du combat des Lumières et du penser par soi-même contre ce qui aliène l'homme : Simondon, lui, faisait au contraire des Lumières - et même de l'Humanisme - ce qui réside en un tel combat et qui, de ce 
fait, peut toujours prendre une nouvelle forme, s'il est vrai que les formes de l'aliénation à combattre sont elles-mêmes toujours nouvelles ${ }^{11}$.

On remarquera d'ailleurs l'ambiguïté du discours de Sloterdijk, qui thématise le psychopouvoir à travers les «médias de la désinhibition » que sont « la télévision, le cinéma de violence » tout en mettant en réalité l'accent sur ces nouvelles formes de bio-pouvoir que sont les technologies génétiques et autres « anthropotechniques » récentes. S'il y a bien une ambiguïté, c'est parce que le discours sur le bio-pouvoir et celui sur le psychopouvoir, que Sloterdijk semble vouloir articuler, n'ont ni le même ancrage théorique, ni les mêmes visées: si Foucault et Stiegler sont irréconciliables, c'est parce que la maîtrise spatiale des corps et la maîtrise temporelle des esprits n'ont pas les mêmes conditions de possibilité et ne font pas peser sur nous les mêmes dangers. Stiegler le dit à sa manière en reprochant à Foucault de ne pas avoir pensé la "prothéticité " originelle de l'esprit humain, qui seule rend possible l'actuelle « désindividuation » par des «rétentions tertiaires»-publicités, films, jeux vidéo - devenues nocives ${ }^{12}$. Les analyses à venir, qui porteront sur autre chose, feront toutefois aussi comprendre que Sloterdijk, tout en thématisant le psycho-pouvoir et ses conditions prothétiques originelles, reste un penseur de l'espace plutôt que du temps. Sans doute est-ce la raison pour laquelle, chez lui, ni le recours aux travaux paléo-anthropologiques ni le discours sur les « médias de la désinhibition » ne parviennent à devenir centraux.

Enfin - et avant d'en venir désormais à mon propos central et donc à une seconde partie de l'analyse -, je crois qu'au vu du scandale qu'il a provoqué, on pourra tout au moins reprocher à Sloterdijk d'avoir été soit particulièrement maladroit, soit particulièrement provocateur à des fins de médiatisation - je penche personnellement pour la seconde hypothèse, s'il est vrai que Sloterdijk est tout sauf naïf.

\section{L'habiter comme co-constitution de l'homme et du Monde}

Dans ses entretiens avec Heinrichs, qui sont intitulés Ni le soleil ni la mort en référence à la fameuse formule de La Rochefoucauld, le philosophe-écrivain allemand revient sur un tel « malentendu » à partir de la thématique qui va m'intéresser ici : la thématique de l'« habiter » telle qu'il la développe dans son grand œuvre, intitulé Sphères. Il répond en effet à Heinrichs :

Selon ma conviction, le devenir-humain en général, et l'ouverture de la clairière en particulier, ont quelque chose à voir avec la domestication, c'est-à-dire avec la domiciliation de l'homo sapiens. Le devenir-humain a été, en tant que tel, un acte d'élevage spontané de soi-même. [...] On peut ainsi comprendre pourquoi une querelle permanente pour l'éducation et le dressage de l'être humain parcourt les cultures avancées. [...] La métaphore du «parc humain » est donc une paraphrase de Platon - j'associais moi-même au "parc» des significations plus américaines qu'européennes. [...] Mais peut-être devrait-on avoir une réflexion positive et se dire que le scandale était un bon signe. [...] L'un des premiers résultats de cette controverse est en tout cas le fait que l'on a commencé à comprendre comment l'affaire de ce que l'on appelle les « anthropotechniques » doit forcément devenir l'objet d'une anthropo-politique. [...] - c'est un état de fait auquel Michel Foucault a déjà fait allusion il y a vingt-cinq ans, dans ses réflexions sur ce qu'il appelait la « biopolitique.$^{13}$ 
Où l'on retrouve bien la référence à Foucault, même si jusqu'à son récent ouvrage $T u$ dois changer ta vie - où Foucault est assez présent - les sources privilégiées de Sloterdijk étaient plutôt - et entre autres évidemment - Friedrich Nietzsche, Gabriel Tarde, Martin Heidegger bien sûr, Gaston Bachelard, Jacques Lacan, Gilles Deleuze, Elias Canetti, Jacques Derrida, Bruno Latour ${ }^{14}$. La référence centrale à Heidegger transparaît du reste aux premières lignes du passage, lorsque Sloterdijk pense à nouveaux frais «l'ouverture de la clairière ", c'est-à-dire l'être-au-monde du Dasein comme « là » de l'« être » : dans Règles pour le parc humain déjà, il reprochait à Heidegger de ne pas avoir compris que l'« analytique du Dasein » doit être réécrite à partir de la paléo-anthropologie :

le séjour humain dans la clairière - en termes heideggériens, le fait que l'homme se tienne ou soit tenu dans la clairière de l'Être - ne constitue nullement un rapport ontologique primitif qui ne serait accessible à aucun autre questionnement. Il existe une histoire, résolument ignorée par Heidegger, de la sortie de l'être humain dans la clairière - une histoire sociale de la manière dont l'homme peut être touché par la question de l'Être, et une mobilité historique dans l'ouverture béante de la différence ontologique. ${ }^{15}$

16 En d'autres termes, si l'homme est cet étant qui s'interroge sur son être, c'est parce qu' une histoire l'a conduit à se tenir dans cette «clairière de l'Être ». Heidegger avait donc eu tort, dans Sein und Zeit, de vouloir penser d'abord l'être-au-monde du Dasein et ne penser ensuite le vivant animal que "par privation» à partir du Dasein ${ }^{16}$ : c'est bien plutôt le mouvement inverse que doit accomplir la pensée. Ce propos est poursuivi et précisé dans La Domestication de l'Être, où Sloterdijk écrit :

si l'on veut s'en tenir à l'alliance avec Heidegger, penseur de l'extase existentielle, il faut parallèlement se décider à mettre entre parenthèses le refus affecté manifesté par Heidegger à l'égard de toutes les formes d'anthropologie empirique et philosophique, et expérimenter une nouvelle configuration entre l'ontologie et l'anthropologie. Il s'agit à présent de comprendre que même la situation fondamentale et apparemment irréductible de l'être humain, qui porte le nom d'être-au-monde et se caractérise comme l'existence ou comme le fait de se tenir à l'extérieur dans la clairière de l'Être, constitue le résultat d'une production dans le sens originel du terme [...]. Je demande donc, pensant avec Heidegger contre Heidegger, comment l'homme est venu à la clairière, et comment a été produit l'éclair à la seule lumière duquel le monde, en tant que monde, a pu commencer à briller. ${ }^{17}$

On remarquera que si l'analytique du Dasein doit donc être réécrite à partir de la paléoanthropologie, réciproquement il s'agit bien de « s'en tenir à l'alliance avec Heidegger, penseur de l'extase existentielle", parce qu'aux yeux de Sloterdijk aucune anthropologie empirique ou "positive " ne rendra compte de cette "ex-stase » qui singularise l'homme, c'est-à-dire de ce fait étrange qu'est l'extériorité de l'esprit à luimême, constitué qu'il est par un "monde » qui n'est pas un simple «milieu», et qui n'est plus naturel mais technique.

Le pari de Sloterdijk est donc en réalité de penser entre l'anthropologie empirique, qui ignore l'extase propre à l'homme, et l'ontologie philosophique «à la Heidegger », qui ignore le devenir-humain, et c'est à cette fin que se développe la théorie métaphorique que Sloterdijk baptise "sphérologie", et qui consiste à penser le devenir-homme objet de l'anthropologie - à partir de la problématique de l' habiter - comme problématique en même temps ontologique car rendant compte de la «clairière de l'Être ». Ainsi écrit-il dans « La Domestication de l'Être » : 
Nous utilisons les métaphores ontologiques de l'habitat dans la maison de l'Être comme les indicateurs du mouvement de pensée anthropologique, et nous demandons par conséquent de quelle manière un être vivant encore tout à fait préhumain [...] s'est quant à lui mis sur la route qui mène dans la maison de l'Être. La réponse réside pour sa plus grande part dans la métaphore elle-même, dès que l'on suspend ses significations transposées et que l'on se représente le devenir-humain lui-même comme une véritable affaire domestique, comme un drame de la domestication, dans une acception inhabituellement large de ce terme. [...] L'analytique de la maison ou du lieu primitif est par conséquent la véritable épreuve de viabilité pour cette nouvelle constellation théorique formée par «l'Être et l'Espace ». Le concept d'espace qui entre ici en jeu est manifestement un concept non physique et non trivial [...] Dans des travaux récents, j'ai proposé pour cet espace non-trivial l'expression de "sphère ", et j'ai tenté de montrer en détail comment l'on peut penser l'extension ou l'aménagement originel des dimensions à l'intérieur de cette sphère. [...] Avec le concept de sphères, on comble une faille dans le champ conceptuel fondamental des théories de l'espace, une faille qui s'ouvrait [...] entre le concept d'environnement et le concept de monde [...]. ${ }^{18}$

Ce passage décisif permet de comprendre que si la notion de sphère doit réconcilier l'anthropologie empirique, pour laquelle il n'existe que des environnements ou des " milieux », et l'ontologie philosophique, pour laquelle après Heidegger il faut rendre compte de l'être-au-monde de l'homme, c'est parce que cette notion de sphère ne relève ni de l'anthropologie empirique ni de l'ontologie philosophique, que cette dernière soit d'ailleurs entendue en son sens heideggérien ou non : aux yeux de Sloterdijk, la notion de sphère relève quasiment d'une nouvelle discipline - la dite "sphérologie » - qui pense non plus avec des concepts mais avec des métaphores, parce qu'il s'agit pour lui de penser ce qui n'est pas objet de connaissance proprement dite - ce en quoi la sphérologie hérite d'ailleurs encore, en réalité, de la philosophie, dont elle pourrait à ce titre sembler être la réalisation par passage dans son "Autre», métaphorique et non plus conceptuel.

Dans le même temps, la sphère est aussi un entre-deux génétique - au sens de la genèse - entre l'« environnement» de l'animal et le "Monde» de l'homme: elle désigne d'abord chez Sloterdijk cette relation entre deux êtres - qui en sont les "pôles", dit-il - par laquelle l'environnement va devenir ce qu'il faudra habiter afin que puisse par là se dessiner un Monde. C'est donc cette notion de sphère qui fait le pont entre le rapport de l'animal à son milieu et le rapport de l'homme à son Monde. Sloterdijk précise ici que la nouvelle «analytique existentiale » ainsi permise après Heidegger serait donc une " analytique de la maison ou du lieu primitif », à la condition cependant d'entendre la notion de "maison », comme celle de " domestication », «dans une acception inhabituellement large ", c'est-à-dire en réalité dans le sens de la "maison de l'Être »: habiter, c'est ici simplement dépasser le rapport de l'animal à son milieu pour créer un monde par lequel l'homme devient homme, c'est-à-dire par lequel il devient cet étant qui s'interroge sur son être.

Deux remarques, ici :

- On aura compris que contrairement à Jacob von Uexküll, Sloterdijk ne distingue pas l'environnement de l'animal et le «milieu de vie» (Umwelt) de l'animal, car ce qui compte à ses yeux c'est le fait que de toute façon chez l'animal il n'y a pas d'« être-aumonde ». Ce qui, chez Sloterdijk, et comme nous le verrons bientôt plus précisément, est à comprendre au sens où seul l'homme porte en lui-même la capacité à élargir la "sphère " de l'intime: chez l'animal, le milieu de vie (Umwelt) ne se distingue de l'environnement 
que comme une intimité restrictive, tandis que la genèse simultanée de l'homme et du Monde pensée par Sloterdijk est le processus par lequel la sphère de l'intime s'étend - ce que Sloterdijk nommera bientôt « expansion du psychique »;

- On pourra penser ici également au passage de Du mode d'existence des objets techniques dans lequel Simondon soutient que «juste au-dessus » de la relation « du vivant à son milieu » se tient le rapport de l'homme à l'« unité magique primitive» du monde, laquelle rendra ensuite possible le rapport technique au monde -comme du reste le rapport religieux - et donc aussi les habitats - au sens cette fois étroit du terme. Sans doute ce parallèle nous aide-t-il à comprendre que ce que Sloterdijk nomme " habiter " précède en réalité l'habitat en tant qu'artefact, parce que cela relève de la "sphère " comme relation particulière à l'autre, véritable pont entre le rapport à l'environnement et le rapport à un Monde. On remarquera cependant l'ambiguïté qui en résulte, à nouveau, dans la pensée de Sloterdijk, car ici la technique n'est plus originellement constitutive de l'homme qu'en un sens métaphorique, celui de la «domiciliation » prise en son sens «inhabituellement large»: contrairement à Stiegler, ni Simondon ni Sloterdijk ne posent encore, dans cette phase originelle, des artefacts dont la fabrication serait en même temps transformation du primate en homme. Simondon, lui, surmontait cette bizarrerie d'une "phase magique primitive " sans artefacts en disant que de toute façon, sa pensée "génétique » des "phases de la culture " n'était pas une pensée historique du devenir de l'homme: elle serait davantage à penser comme une eidétique génétique, qui dégage en effet des lois d'essences psychiques et constitue ainsi l'esquisse d'une phénoménologie non-dialectique, et génétique plutôt qu'historique, de l'esprit. Or, la distinction subtile entre genèse et histoire n'est pas reprise par Sloterdijk, et c'est pourquoi se pose ici la question de la cohérence globale du propos de ce dernier.

24 Ainsi sommes-nous en tout cas reconduits au passage sur lequel s'ouvrait cette seconde partie de l'analyse, c'est-à-dire le passage où Sloterdijk introduisait les idées de «domestication» et de "domiciliation» de l'être humain. Mais nous le sommes désormais avec la conscience que ce qui se joue en vérité au fondement de la "sphérologie» de Sloterdijk, c'est une nouvelle forme de pensée de l'intersubjectivité, puisque le philosophe allemand, initialement formé à la phénoménologie, fait de la relation à l'autre le lieu originel - ce qui est à prendre en son sens spatial mais prégéométrique - de l'« habiter » comme co-constitution de l'homme et du Monde. C'est ce que nous fait définitivement comprendre Sloterdijk lorsqu'au seuil du troisième volet de Sphères, qui est intitulé Écumes, il résume l'entreprise intellectuelle des deux premiers volets de sa trilogie :

Sphère I propose une description (parfois nouvelle, selon l'auteur) de l'espace humain et souligne que la coexistence de proximité entre des êtres humains crée un intérieur d'une nature particulière. Nous lui donnons le nom de microsphère et nous le caractérisons comme un système immunitaire de l'espace psychique, système très sensible et accessible à l'apprentissage. On souligne la thèse selon laquelle le couple constitue une dimension plus réelle que l'individu - ce qui signifie aussi que l'immunité du Nous constitue un phénomène plus profond que l'immunité $\mathrm{du}$ Moi. [...] Nous caractérisons les univers de proximité humains comme des espaces surréels pour exprimer l'idée que même des rapports dépourvus de dimension spatiale comme la sympathie et la compréhension se transposent dans des relations quasi-spatiales pour devenir concevables et vivables. [...] Dans Sphères II, on tire les conséquences de la compréhension de la nature ek-statique et surréelle de l'espace vécu et habité. Cela se produit sous la forme d'un grand récit sur l'expansion du psychique. On pourrait donner à l'entreprise le nom de roman philosophique. ${ }^{19}$ 
La notion de « roman philosophique » ici revendiquée par Sloterdijk désigne bien ce qui s'offre, chez lui aussi, comme une nouvelle phénoménologie de l'esprit, dont le caractère dialectique se maintient sous la forme atténuée d'un simple dualisme fondamental: Sloterdijk, ainsi qu'il l'explique dans les entretiens avec Heinrichs, s'oppose à toute la tradition substantialiste - mais aussi au monisme qui l'accompagne - en posant que «la sphère n'est, depuis le commencement, donnée que sous une forme dyadique, comme structure de la bi-unité $»^{20}$.

\section{De la « sphérologie » à l'herméneutique historico- topologique de la philosophie}

Dans Écumes, Sloterdijk poursuit et radicalise ce «roman philosophique » en tant que pensée à la fois ontologique et paléo-anthropologique de la venue au Monde, pensée qui se substitue d'un côté aux pensées scientifiques mais non unifiées de la "venue au monde " comme simple naissance - phénomène à la fois biologique, psychologique, sociologique -, et d'un autre côté à la pensée heideggérienne du simple être-au-monde, qui n'est pas encore une genèse de l'homme et du Monde. Le paradoxe étant alors que la pensée génétique de la venue au Monde, par laquelle Sloterdijk se distingue de Heidegger, est une pensée de l'Être et l'Espace qui remplace la pensée heideggérienne de l'Être et le Temps. Ce paradoxe se résout si l'on remarque que chez Heidegger, la primauté de la notion de temps tenait au fait que le temps était "sens de l'être », et ce fait restait étranger à l'idée qu'il faudrait inscrire l'être-au-monde dans la temporalité d'une «venue au Monde ». La question qui subsiste, toutefois, est celle de savoir si la pensée de l'Être et l'Espace chez Sloterdijk se donne véritablement les moyens de dégager les types de temporalité qui se rattachent aux divers stades de la genèse sphérologique comme « expansion du psychique».

Mais c'est sur une autre question que je voudrais m'arrêter ici, dans la lignée des réflexions préalables sur l'« habiter » comme co-constitution de l'homme et du Monde, et pour conclure cette toute première étude que je consacre à Sloterdijk. Voici donc le point annoncé : au chapitre II d'Écumes, Sloterdijk en vient à penser «l'architecture moderne comme explicitation du séjour $»^{21}$. Sa thèse s'exprime dans un passage qu'il faut citer en entier malgré sa longueur, parce que c'est en lui aussi que se formule et s'explicite l'étrange idée qui fait mon titre même : cette étrange idée selon laquelle la "révolution de l'espace » liée à l'architecture du xxe siècle fait de cette dernière une « réalisation de la philosophie »...

Voici en effet ce qu'il écrit :

La véritable "révolution de l'espace» $\mathrm{du} \mathrm{xx}^{\mathrm{e}}$ siècle est l'explicitation du séjour humain ou de la demeure dans un intérieur [...]. La définition de l'architecture de la modernité en découle: elle est le media dans lequel s'exprime progressivement l'explicitation du séjour humain dans des intérieurs faits par l'homme. Par conséquent, l'architecture constitue depuis le $\mathrm{xIX}^{\mathrm{e}}$ siècle quelque chose que l'on aurait appelé, dans la période du Vormärz, une "réalisation de la philosophie ». Pour employer encore une fois les mots de Heidegger : elle met en œuvre la Er-örterung, la localisation du Dasein. Elle ne se contente pas d'être l'exécutrice, plus ou moins consciente de son savoir-faire, de l'activité d'habitat de l'être humain, dont on peut remonter les traces jusqu'au premier arrangement de campements, de grottes et de cabanes. Elle reformule les "lieux" où peut se dérouler quelque chose comme l'habitat, la demeure et l'être-chez-soi de groupes et d'individus dans des conditions 
où la référence à soi-même est notable, où l'argent joue un rôle important d'intermédiaire, où la législation, la connexion et la mobilisation ont atteint un degré élevé. De ces lieux, nous savons qu'on ne peut plus les penser seulement comme l'ici et le là-bas d'un «monde de la vie ». Un lieu, dans les conditions en vigueur, c'est un quantum d'air réaménagé et conditionné, un local d'atmosphère transmise et actualisée, un nœud de relations hébergées, un carrefour dans un réseau de flux de données, une adresse pour initiatives d'entrepreneurs, une niche pour les relations à soi-même, un camp de base pour des expéditions dans l'environnement du travail et de l'expérience, un site pour des affaires commerciales, une zone de régénération, un garant de la nuit subjective. Plus l'explicitation progresse, plus l'édification de logements ressemble à l'installation de stations spatiales. $^{22}$

28 Je disais plus haut que la sphérologie était pour ainsi dire une nouvelle discipline qui, dans l'esprit de Sloterdijk, "réalisait la philosophie » en lui permettant de se dépasser dans son Autre, métaphorique et non plus conceptuel. Or, le passage cité ici exprime une idée autre, dont il faut examiner la compatibilité avec la première : l'architecture $\mathrm{du} \mathrm{XIX}^{\mathrm{e}}$ siècle a accompli une "révolution de l'espace » par laquelle c'est l'architecture elle-même qui s'offre comme "réalisation de la philosophie ». Comment comprendre cette idée de « réalisation »? Sloterdijk donnait deux précisions :

- d'abord, l'architecture ne se contente pas d'exécuter l'habitat en appliquant une théorie de l'habitat, elle reconfigure l'espace lui-même et donc le « séjour humain » sur Terre. Pour autant, l'architecture pourrait, ce faisant, rester l'exécutrice de la philosophie, comprise comme conception fondamentale du "séjour humain " qui demanderait à s'appliquer via les théories de l'habitat puis l'architecture ;

30 - ensuite, l'idée de « réalisation de la philosophie » doit être comprise, ajoute Sloterdijk, au sens qu'on lui donnait durant le Vormärz, c'est-à-dire cette période allemande qui s'étend de 1815 à 1849 et qui fut marquée, dans le domaine des idées, d'un côté par Hegel et Marx et leur idée de « réaliser la philosophie » dans la Science suprême comme dans l'Histoire concrète des hommes - ce qui donne déjà un double sens à l'idée de " réalisation»-, d'un autre côté par ce courant littéraire allemand - influencé par Heinrich Heine - auquel fut donné le nom de «Jeune Allemagne », et qui faisait primer la révolution politique sur les visées esthétiques.

31 La synthèse de ces deux précisions données par Sloterdijk débouche dans un premier temps sur l'idée suivante: l'architecture contemporaine est ce qui permet à la philosophie, elle-même "réalisée » sur le plan théorique en "sphérologie " grâce à l'œuvre de Sloterdijk, de se réaliser dans l'Histoire concrète des hommes. C'est ce double mouvement que résume Sloterdijk dans ses entretiens avec Heinrichs :

Dans mes recherches sur l'art de la constitution d'espaces et d'atmosphères, je me suis heurté au fait que les experts qui, pour des motifs professionnels, ont le plus souvent affaire à l'espace habitable, animé, les architectes modernes, sont simultanément ceux qui ont développé les sphérologies techniques les plus lucides. J'ai parfois été perplexe en découvrant de quelle manière explicite certains d'entre eux avaient depuis longtemps pensé et mis en œuvre ce que j'avais cherché dans mes mouvements de quête, qui avaient duré plusieurs années. Je pense à certains ensembles réellement construits, mais plus encore à des maquettes et projets graphiques, jusqu'aux architectures poétiques, picturales et spéculatives conçues depuis le début $\mathrm{du} \mathrm{xx}^{\mathrm{e}}$ siècle, à l'ère de l'expressionisme aussi bien que dans les vagues du constructivisme, et qui vont jusqu'à notre époque - pensez aux croquis et aux travaux de Bruno Taut, aux projets de Tatline et Melnikov, aux fantasmes spatiaux graphiques et picturaux de Finsterlin - pour ne citer ici que quelques-uns 
des pionniers. Leurs impulsions sont reprises aujourd'hui par les tenants de la construction biomorphe assistée par ordinateur..$^{23}$ contemporaine vis-à-vis de la philosophie « réalisée » en sphérologie s'offre comme le complément d'une autre thèse peut-être encore plus étonnante : la thèse selon laquelle l'histoire de la philosophie n'est que l'histoire des "projections" conceptualisées de la sphérologie intime et inconsciente des penseurs. Ainsi par exemple, le second volet de Sphères, intitulé "Globes ", avait tâché de montrer que toute la pensée théologique et cosmologique dite "métaphysique ", jusqu'à Leibniz et même Hegel, n'est que la projection conceptualisée, sur Dieu et sur le Monde compris comme des totalités, d'une sphérologie intime et encore naïve parce que pensant la sphère comme un globe parfait et déjà donné, au lieu de reconduire toute totalité à l'intimité des toutes premières «sphères » par lesquelles commencent de se dessiner à la fois un sujet pensant et un Monde: les «sphères» de l'être-ensemble à deux, trois, etc. Le dernier mot de l'entreprise de Sloterdijk serait alors un mot herméneutique appliqué à la philosophie ellemême. Ce mot, le voici : «Je prophétise un autre passé à la philosophie » ${ }^{24}$.

\section{BIBLIOGRAPHIE}

Barthélémy Jean-Hugues, "What new Humanism today ?", Cultural Politics, C. Turner (trad.), vol. 6, $\mathrm{n}^{\circ}$ 2, Berg Publishers, 2010, p. 237-252. La version française est disponible à l'adresse : http:// www.canal-u.tv/video/universite_de_tous_les_savoirs/ quel_nouvel_humanisme_aujourd_hui_jean_hugues_barthelemy.4112

Barthélémy Jean-Hugues, "Individuation and knowledge. The refutation of idealism in Simondon's Heritage in France”, SubStance, M. Hayward \& A. De Boever (trad.), vol. 41, n³, University of Wisconsin Press, 2012.

Heidegger Martin, Sein und Zeit [Halle, 1935], Max Niemeyer Verlag, Tübingen, 1927.

Sloterdijk Peter, Ni le soleil ni la mort, O. Mannoni (trad.), Paris, Hachette Littératures, 2003.

Sloterdijk Peter, Écumes, O. Mannoni (trad.), Paris, Hachette Littératures, 2005.

Sloterdijk Peter, « La Domestication de l'Être », in Règles pour le parc humain, O. Mannoni (trad.), Paris, Mille et une nuits, 2010.

Sloterdijk Peter, Règles pour le parc humain, O. Mannoni (trad.), Paris, Mille et une nuits, 2010.

Stiegler Bernard, La technique et le temps 1 : «La faute d'Epiméthée », Paris, Galilée, 1994.

Stiegler Bernard, Prendre soin. De la jeunesse et des générations, Paris, Flammarion, 2008.

\section{NOTES}

1. Peter Sloterdijk, Règles pour le parc humain, O. Mannoni (trad.), Paris, Mille et une nuits, 2010 (réédition). 
2. Ibid., p. 52.

3. Peter Sloterdijk, « La Domestication de l'Être », in Règles pour le parc humain, p. 86.

4. Ibid., p. 85-86.

5. Où l'on comprend pourquoi Sloterdijk déclare: «si je voulais rassembler du matériau autobiographique sur mes débuts, je devrais d'abord et avant tout citer des noms comme Adorno et Bloch, que j'ai entièrement absorbés pendant mes études bien que la trace de leur influence dans mon travail ne soit plus qu'indirectement décelable », Peter Sloterdijk, Ni le soleil ni la mort, O. Mannoni (trad.), Paris, Hachette Littératures, 2003, p. 16. Comme Stiegler dans le premier tome de La technique et le temps, Sloterdijk, depuis Règles pour le parc humain et La domestication de l'Être, pense non seulement le devenir-homme sur fond de travaux paléo-anthropologiques et dans un dialogue avec l'analytique du Dasein de Heidegger, mais aussi ce qu'Adorno et Horkheimer ont nommé dès 1948 les «industries culturelles ", et la centralité de ces dernières dans le nouveau capitalisme. À la différence de Stiegler, comme on va le voir, Sloterdijk se voudra cependant davantage ce qu'il nommera un "média d'ambiance ", c'est-à-dire une caisse de résonance pour l'esprit du temps, qu'un «média d'urgence » et un intellectuel engagé. Sans doute la nuance sur laquelle s'achevait le passage précédemment cité - et par laquelle était rappelé le fait qu'Adorno ne parle plus à travers Sloterdijk - est-elle en pleine cohérence avec cette différence entre Sloterdijk et Stiegler.

6. Voir mon article "Individuation and knowledge. The refutation of idealism in Simondon's Heritage in France", SubStance, M. Hayward \& A. De Boever (trad.), vol. 41, n 3, University of Wisconsin Press, 2012.

7. Voir mon article "What new Humanism today ?", Cultural Politics, C. Turner (trad.), vol. 6, nº 2, Berg Publishers, 2010, p. 237-252. La version française est disponible à l'adresse: http:// www.canal-u.tv/video/universite_de_tous_les_savoirs/

quel_nouvel_humanisme_aujourd_hui_jean_hugues_barthelemy.4112

8. Peter Sloterdijk, Ni le soleil ni la mort, p. 17.

9. Ibid., p. 13.

10. Ibid., p. 17-18.

11. Sur ce point, voir mon article précédemment cité : « What new Humanism today?».

12. Voir sur ce point Bernard Stiegler, Prendre soin. De la jeunesse et des générations, Paris, Flammarion, 2008.

13. Peter Sloterdijk, Ni le soleil ni la mort, p. 69-70.

14. La moitié seulement de ces références sont des références de Stiegler, qui reprocherait sans doute à Sloterdijk de se vouloir un philosophe-«écrivain» et d'abuser des métaphores pour construire un « roman philosophique », selon l'expression de Sloterdijk lui-même.

15. Peter Sloterdijk, Règles pour le parc humain, p. 36.

16. Voir sur ce point le $\$ 10$ de Sein und Zeit. Martin Heidegger, Sein und Zeit, Max Niemeyer Verlag, Tübingen, 1927.

17. Peter Sloterdijk, "La Domestication de l'Être", in Règles pour le parc humain, p. 86-87. La formule «penser avec Heidegger contre Heidegger » est le titre d'un opuscule de Habermas, que Sloterdijk reprend ici sans le dire. Rappelons que Habermas est à la fois le penseur qui signala l'importance du premier grand livre de Sloterdijk - sa Critique de la raison cynique - et le « gardien de la mémoire » qui fustigea les usages terminologiques de Sloterdijk dans Règles pour le parc humain. Le rapport de Sloterdijk à ce philosophe majeur est donc complexe, et ce d'autant plus que Sloterdijk, ainsi que je l'ai rappelé, a d'abord été nourri de la pensée de l'École de Francfort.

18. Ibid., p. 113-116 (c'est moi qui souligne).

19. Peter Sloterdijk, Écumes, O. Mannoni (trad.), Paris, Hachette Littératures, 2005, p. 9-11 (c'est moi qui souligne).

20. Peter Sloterdijk, Ni le soleil ni la mort, p. 173.

21. Peter Sloterdijk, Écumes, p. 443 (titre du premier sous-chapitre). 
22. Ibid., p. 445-446 (c'est moi qui souligne).

23. Peter Sloterdijk, Ni le soleil ni la mort, p. 296-297 (c'est moi qui souligne).

24. Ibid., p. 245.

\section{RÉSUMÉS}

La pensée de Sloterdijk revisite l'analytique existentiale heideggérienne à partir de la paléoanthropologie, afin de montrer que l'« être-au-monde » propre à l'homme résulte d'une genèse "anthropotechnique», laquelle seule peut éclairer l'impact actuel des «médias de la désinhibition ». Cependant, la «sphérologie » de Sloterdijk ne dépasse conjointement - pour les unifier - l'ontologie philosophique et l'anthropologie empirique qu'en se faisant «roman philosophique » ou théorie métaphorique de l'« habiter " comme "domestication », et revisite pour ce faire la thématique phénoménologique de l'intersubjectivité en définissant la "sphère » minimale comme cette relation entre deux êtres par laquelle l'environnement va devenir ce qu'il faudra habiter afin que puisse par là se dessiner un Monde. L'audace suprême de Sloterdijk est alors de soutenir dans Écumes que l'architecture contemporaine, dans sa spécificité de « révolution de l'espace », a valeur de « réalisation de la philosophie »...

\section{INDEX}

Mots-clés : anthropotechnique, architecture, domestication, être-au-monde, sphérologie 